\title{
Investigation of Waste Tea Leaves (Black) as a Source for a Potential Colouring Agent for Textile Substrates for the Sri Lankan Textile Industry
}

\author{
Wijayapala S. \\ Department of Textile and Clothing Technology, Faculty of Engineering, \\ University of Moratuwa, Katubedda, Sri Lanka \\ *samu@uom.lk
}

\begin{abstract}
Until the invention of synthetic dye "Perkin Mauve" (1856), people used natural sources like stem, bark, leaves of plants etc and for dyeing of clothes. In todays context people are concerned about the harmful effect of using synthetic dyes and is going for natural dyes instead. This is a result of the stringent environmental standards imposed by many countries in a response to the toxic and allergic reactions associated with synthetic dyes. The textile industry is an important economic activity with a $70 \%$ contribution to the Sri Lankan economy. Dyes are an important process requirement for which the country spends about 8.5 mn US\$ annually. Almost all dyes presently used for textile dyeing purposes are of synthetic origin that are imported to the country.
\end{abstract}

Some work on the application of tea on wool, silk jute and cotton are available in the literature (Vankar P.S., 2006). Applications of tea extracts on wool using acetone as the dyeing medium are also reported in the literature. Recently an exhaustive review on the subject of natural dyes in textile applications has been published by Taylor. In this work, natural tea was used to dye cotton fabrics. The colour of the tea leaves was extracted using water as extraction medium. The dyeing was carried out with the dye extracted from waste tea leaves. Washing fastness, light fastness etc, were compared for dyeing carried out using the pre-extracted dye. The effect of mordanting in such dyeing was also investigated and compared.

The present investigation was carried out to revive the old art of dyeing with a natural dye obtained from tea leaves (Camelia sinensis). It belongs to the family Theaceaee, and is commonly known as tea and is a crop plant in Sri Lanka. The dye has good scope in the commercial dyeing of cotton in textile and garment industry. In the present study cotton fabrics were dyed with an aqueous extract of waste tea leaves (black), containing tannins as the main colourant species. The dyeing was carried out without and with metal salts (Copper sulphate) and natural mordants such as Sepalika (Nyctanthes arbor-tristis), Aralu (Ailanthus excelsa). Their fastness properties were investigated. The wash, rub and light fastness of the dyed fabrics were good to excellent. Aqueous extracts of tea yield brown shades with good wash and light fastness on cotton fabrics. All the fastness properties can be further improved by treatment with certain metal salts and bio materials as mordants. A range of attractive colours were produced by the use of extracst of waste tea leaves.

Keywords: Natural dye, Dyeing, Waste tea leaves, Mordanting, Fastness properties 